\title{
The Effect of Coaxial Ring Masses with Different Contact Areas, Mass, and Distribution on Membrane-Type Acoustical Metamaterials' Transmission Loss
}

\author{
Xiao-Ling Gai, Xian-Hui Li, Bin Zhang, Yan-Qi Liu, Peng Xie and Zhi-Hui Ma \\ Beijing Key Laboratory of Environment Noise and Vibration, Beijing Municipal, Institute of Labor Protection, \\ Beijing 100054, China
}

(Received 4 November 2013; accepted 6 October 2014)

The transmission loss (TL) of the membrane-type acoustical metamaterials with coaxial ring masses are investigated using the finite element method. The results show that the TL peak and resonance frequencies of the membrane-type acoustical metamaterials depends on mass, distribution of coaxial ring masses, and the contacting area of coaxial ring masses with the membrane. It is also shown that the coaxial ring masses only affect the TL at low frequencies, while the membrane is effective at all frequencies. Additionally, the double-leaf membrane-type acoustical metamaterials structure has been constructed. The roles of the membrane and ring masses of double-leaf membrane-type acoustical metamaterials structure on TL are investigated. The influence of the depth of air-cavity on the TL is then discussed.

\section{INTRODUCTION}

Low frequency noise has long been regarded as a pernicious form of environmental pollution because it involves blocking large-wavelength waves, which requires thick or heavy materials. ${ }^{1,2}$ In general, it should be a low transmission loss at a low frequency with both thin and lightweight structures. ${ }^{3}$ To overcome this difficulty, composite materials with locally resonant acoustical metamaterials were developed recently. ${ }^{1,4-9}$ Hirsekorn proposed a heuristic model of locally resonant sonic crystals, which allowed one to predict the resonance frequencies in good agreement with the numerical simulations. ${ }^{5} \mathrm{Li}$ and Chan showed the existence of acoustical metamaterials, where the effective density and bulk modulus are both simultaneously negative in the true and strict sense of an effective medium. ${ }^{6}$ A class of sonic shield materials based on the principle of locally resonant microstructures are demonstrated by Ho et al. ${ }^{8}$ One of the main advantages of locally resonant acoustical metamaterials is the ability to prevent sound propagation at low frequencies without the addition of significant mass or modulus bulk. ${ }^{10}$ Large-scale weighted membranes, which are traditionally used in building acoustics, have shown attenuation achieved at varying frequencies. ${ }^{11,12}$ In addition, small-scale membrane-type acoustical metamaterials have been shown to improve sound insulation at low frequencies, surpassing the acoustic mass law by several orders of magnitude over a narrow frequency band. ${ }^{1,13}$ Mei et al. presented a thin-film acoustical metamaterial that comprised of an elastic membrane decorated with asymmetrical rigid platelets that almost reached unity absorption at frequencies where the relevant sound wavelength in the air was three orders of magnitude larger than the membrane thickness. ${ }^{14}$ Using the finite element analysis and experimental techniques, Christina et al. analyzed the transmission loss of membrane-type locally resonant acoustical metamaterials with the added ring masses. ${ }^{10}$
The results showed that the addition of a ring mass to the structure either increased the bandwidth of the transmission loss peak or introduced multiple peaks. This depended on the number of rings, the distribution of mass between the center and ring masses, and the radii of the ring. ${ }^{10}$ Meanwhile, Christina et al. fabricated the arrays of membrane-type acoustical metamaterials and found that the sound transmission at multiple frequencies could be decreased by employing nonuniform mass distribution over the cells in the array. ${ }^{15}$ Ding et al. designed an acoustical metamaterial with multi-band of negative modulus composed of different sized split hollow spheres. ${ }^{16}$ The results indicated that this medium could achieve a negative modulus at a frequency range from 900 to $1500 \mathrm{~Hz} .{ }^{16}$ Although a lot of research has been done on locally resonant acoustical metamaterials, it appears that no relevant reports have been given for acoustical metamaterial of coaxial ring masses with different cross section shapes and double-leaf membrane-type acoustical metamaterials. This paper focuses on the acoustical performance studies of membrane-type acoustical metamaterials of coaxial ring masses with different cross section shapes and double-leaf membrane-type acoustical metamaterials.

In Section 2, sample constructions will be introduced. In Section 3, the transmission loss for membrane-type acoustical metamaterials of coaxial ring masses with four kinds of different cross section shape will be studied. In Section 4, the effect of coaxial ring's mass on transmission loss of membrane-type acoustical metamaterials will be investigated. In Section 5, the effect of coaxial ring distribution on transmission loss of membrane-type acoustical metamaterials will be studied. In Section 6, the main roles of the membrane and coaxial ring $s$ will be discussed. In Section 7, the acoustical performance of the double-leaf membrane-type acoustical metamaterials will be studied. Finally, the conclusions will be given in Section 8 . 


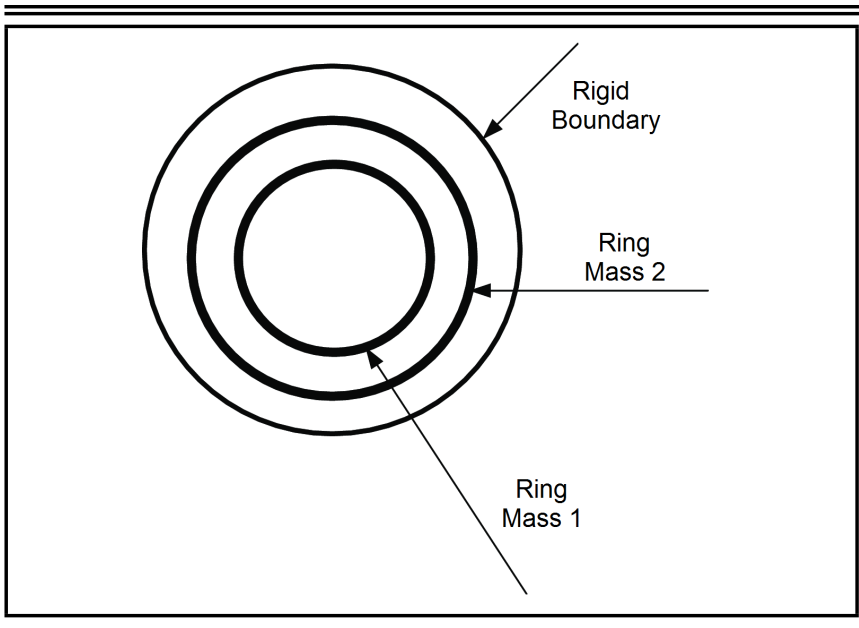

Figure 1. Schematic of membrane-type acoustical metamaterials.

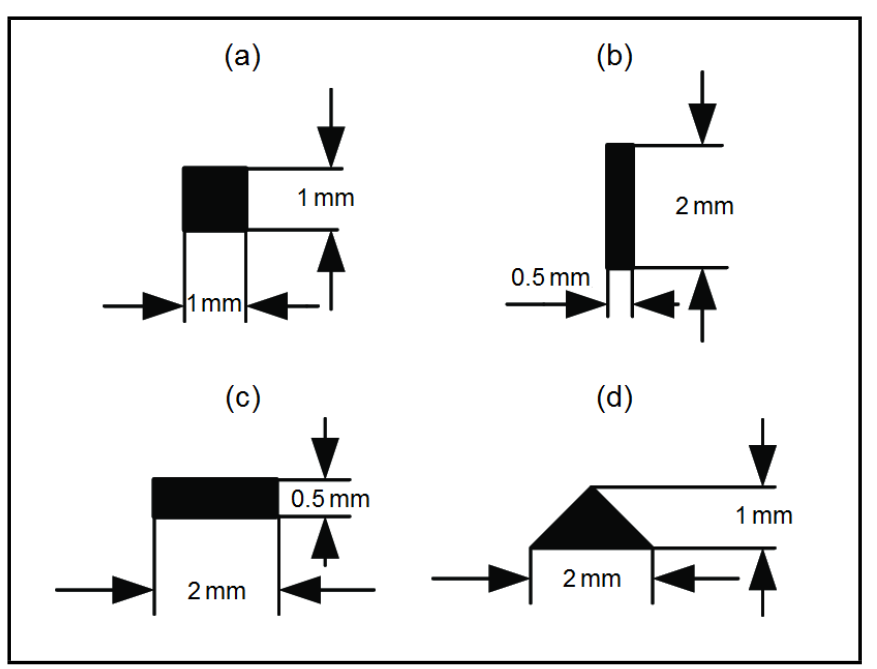

Figure 2. Schematic of four kinds of coaxial ring masses with different cross section shape.

\section{SAMPLE CONSTRUCTION}

Membrane-type acoustical metamaterials of coaxial ring masses with different cross section shape were constructed by using a thin, circular membrane and a two-ring located mass. The membrane that was used was a polyetherimide film, $0.076 \mathrm{~mm}$ thick, and $24 \mathrm{~mm}$ in diameter. The modulus, density, and Poisson's ratio for the membrane material were $6.9 \times 10^{9} \mathrm{~Pa}, 1200 \mathrm{~kg} / \mathrm{m}^{3}$, and 0.36 , respectively. The edge of the membrane was bonded to a rigid support structure. The masses were added to the membranes by attaching two small copper rings. The modulus, density, and Poisson's ratio for the copper material were $110 \times 10^{9} \mathrm{~Pa}, 7800 \mathrm{~kg} / \mathrm{m}^{3}$, and 0.35 , respectively. The total mass of the different shape rings were kept to be invariable. The center of the first ring was located at $r=4.5 \mathrm{~mm}$ and for the second ring, was located at $r=8.5 \mathrm{~mm}$. Figure 1 shows a schematic for membrane-type acoustical metamaterials of coaxial ring masses with different cross section shape. Figure 2 shows four kinds of coaxial ring masses with different cross section shape.

\section{TRANSMISSION LOSS ANALYSIS}

Finite element analysis was used to calculate the transmission loss of membrane-type acoustical metamaterials using
COMSOL software. The 2DS model was used for geometric modeling. An axisymmetric model was used for the analysis to facilitate calculation intensity. In the simulations, structural and acoustical modules were used to create a structuralacoustic interaction program. The acoustical structure boundary excluded the junction surface masses and the membrane in the simulation to make both the masses and the membrane into a whole with different material properties. The membrane was bonded to the support structure, which was a rigid boundary condition is imposed at the edge of the membrane. The mass and membrane were linear elastic materials. The initial pressure magnitude normal incident on the structure was set at $1 \mathrm{~Pa}$. The mass, membrane, and air cavities were modeled with free-triangular mesh elements. The number of degrees of freedom was 7732 .

The nonlinear analysis was not used for the membrane. The masses were added to the membranes by attaching two small rings. Figure 3 shows the four kinds of schematic of the axisymmetric finite element analysis models. Structural and acoustical modules were used in the finite element analysis to create a structural-acoustic interaction program. There were two air cavities on both sides of the membrane-type acoustical metamaterials, which were similar to the impedance tube structure. The membrane, mass ring, and air cavities were modeled with free-triangular mesh elements.

The following equation defines the transmission loss of the membrane-type acoustical metamaterials:

$$
T L=10 \log \frac{W_{i}}{W_{o}} .
$$

Here, $W_{i}$ and $W_{o}$ denoted the incoming power at the inlet and the outgoing power at the outlet, respectively. We calculated each of these equations as an integral over the whole circular disk:

$$
\begin{aligned}
& W_{i}=\int \frac{p_{0}^{2}}{2 \rho c} d L ; \\
& W_{o}=\int \frac{\left|p^{2}\right|}{2 \rho c} d L .
\end{aligned}
$$

Figure 4 shows the transmission loss of membrane-type acoustical metamaterials of coaxial ring masses with four kinds of different cross section shapes. The transmission loss peak corresponded to anti-resonance behavior, where minimal sound was transmitted across the structure, while the transmission loss minima corresponded to the resonance frequencies, where $100 \%$ of the sound was transmitted. In comparing Figs. 4(a) to 4(d), we found that the different cross section shape coaxial ring with the same mass affected the transmission loss peak and resonance frequencies. Figure 4(a) shows the transmission loss for the square cross section coaxial ring masses. The transmission loss profile for this configuration exhibited a low-frequency minimum $(340 \mathrm{~Hz})$, a highfrequency minimum $(4010 \mathrm{~Hz})$, and a transmission loss peak $(2240 \mathrm{~Hz}$ and $78.4 \mathrm{~dB})$. Figures 4(b) and 4(c) show the finite element analysis results of transmission loss for two rectangular cross section coaxial ring masses with side lengths of $0.5 \mathrm{~mm} \times 2 \mathrm{~mm}$ and $2 \mathrm{~mm} \times 0.5 \mathrm{~mm}$. There were two resonance peaks $(280 \mathrm{~Hz}$ and $3550 \mathrm{~Hz}$ ) and transmission loss peaks $(1830 \mathrm{~Hz}, 5515 \mathrm{~Hz})$, when the cross section of the ring was 


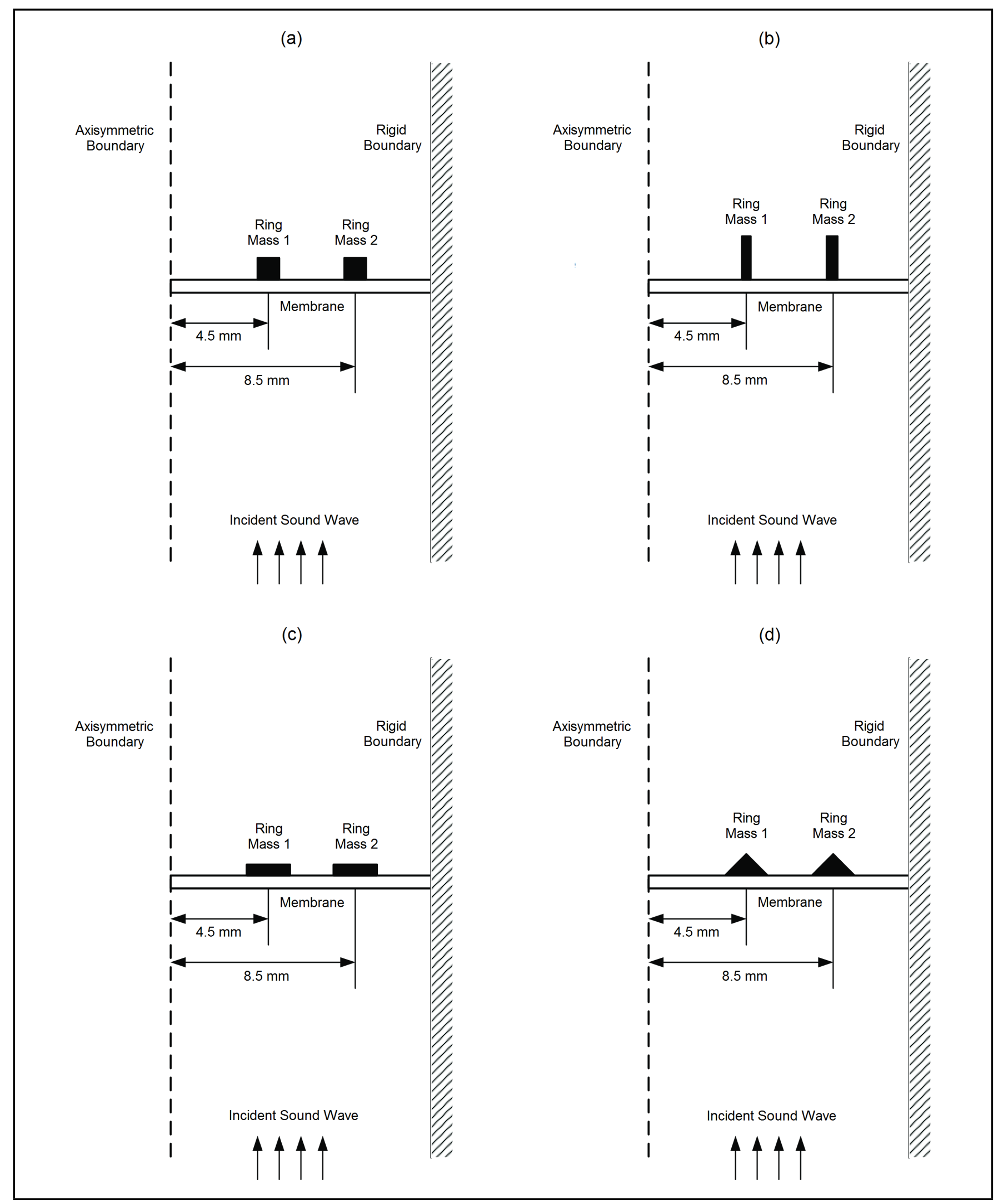

Figure 3. Four kinds of schematic of the axisymmetric finite element analysis models. 
(a)

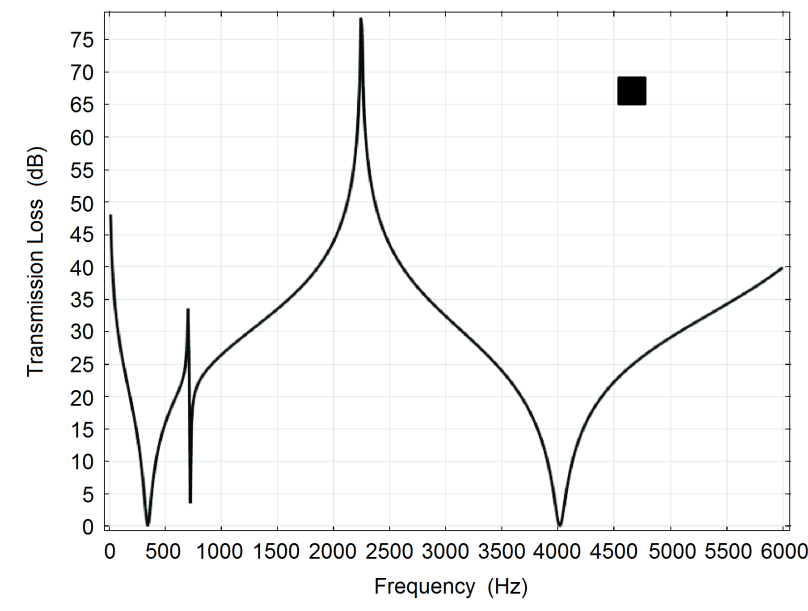

(c)

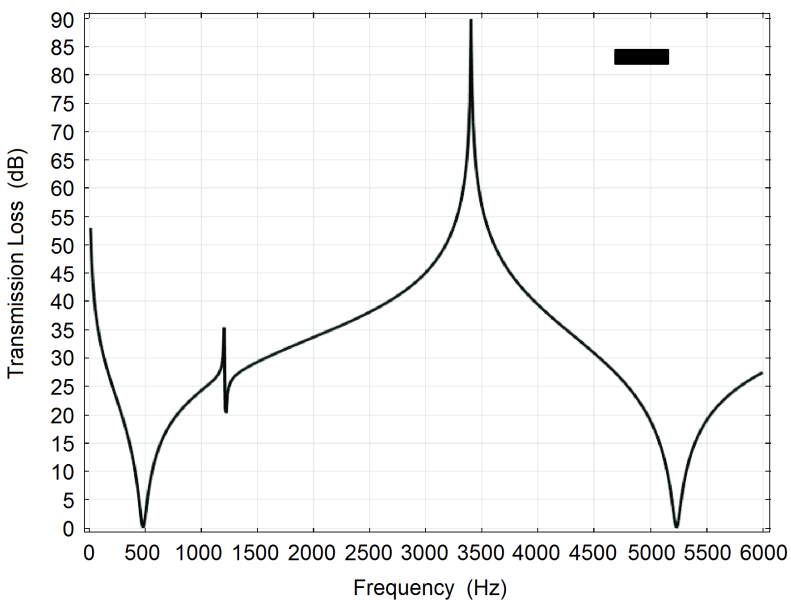

(b)

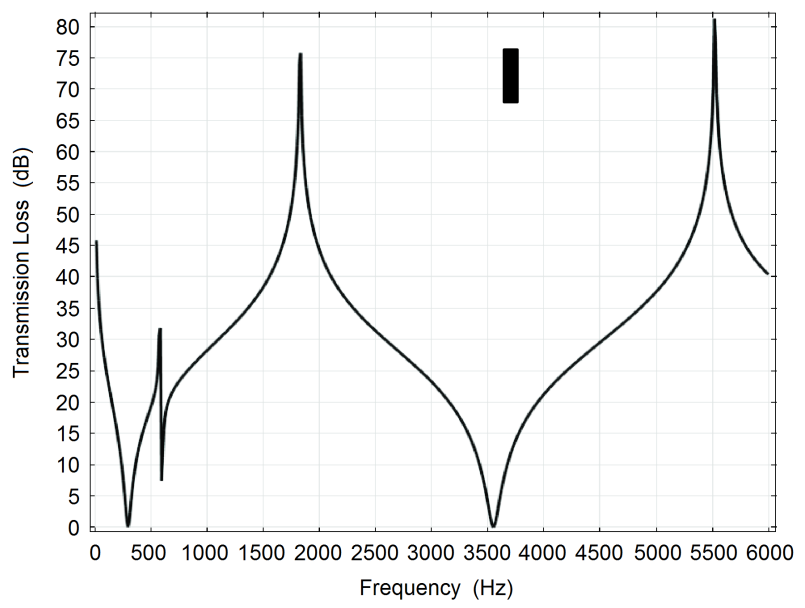

(d)

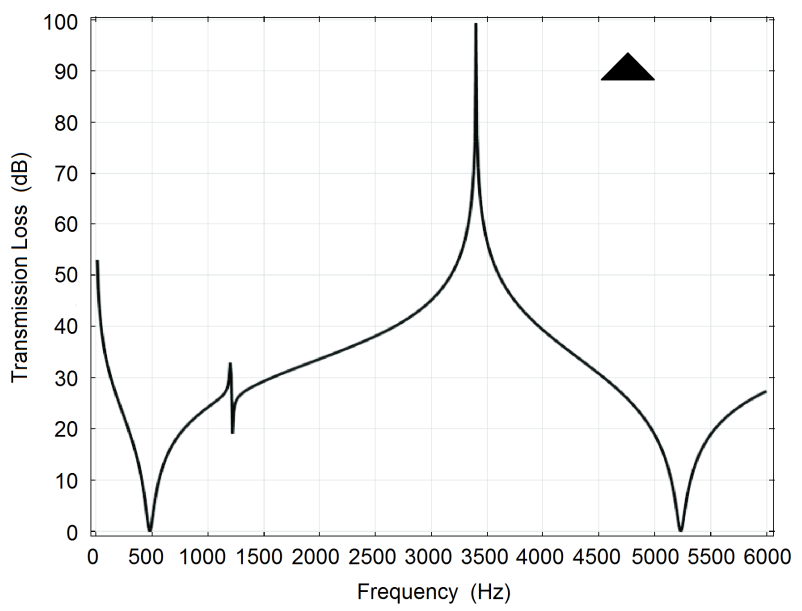

Figure 4. Transmission losses of membrane-type acoustical metamaterials using coaxial ring masses with four kinds of different cross section shape.

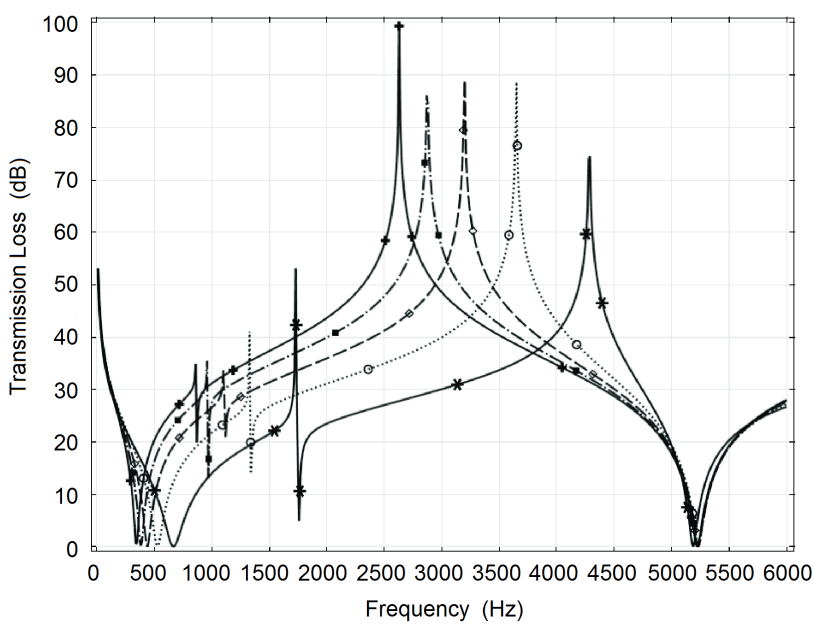

Figure 5. Effect of ring's mass on transmission loss of membrane-type acoustical metamaterials. 
(a)

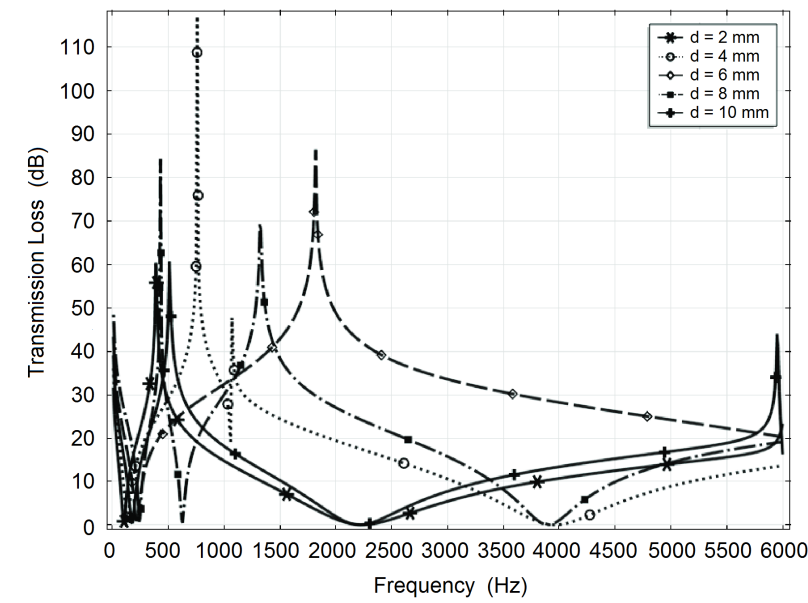

(c)

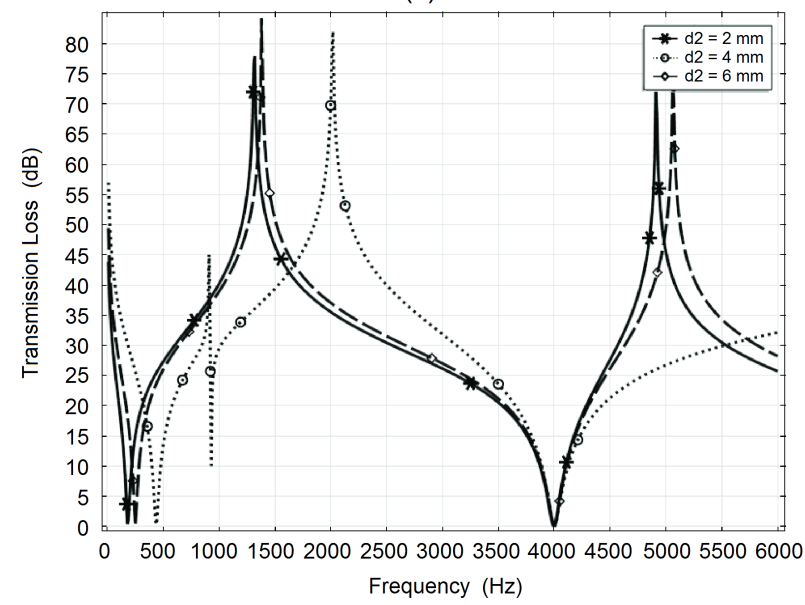

(b)

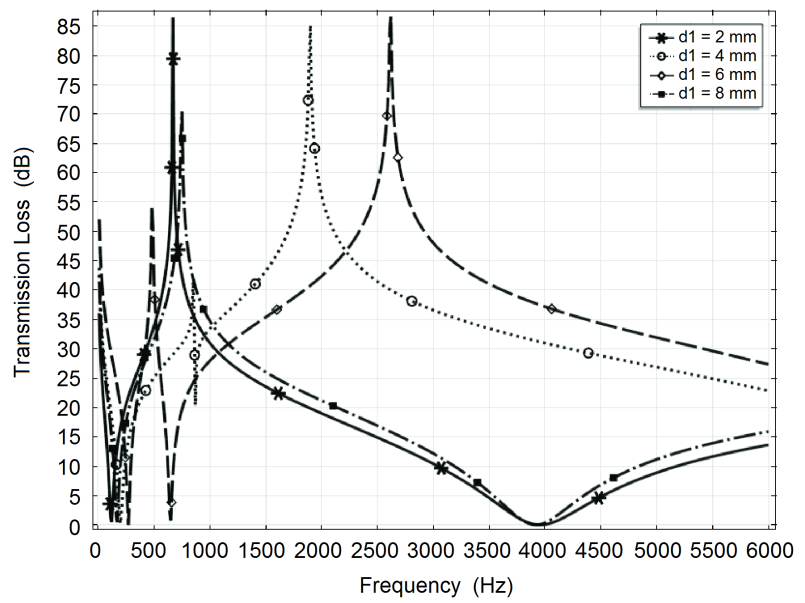

(d)

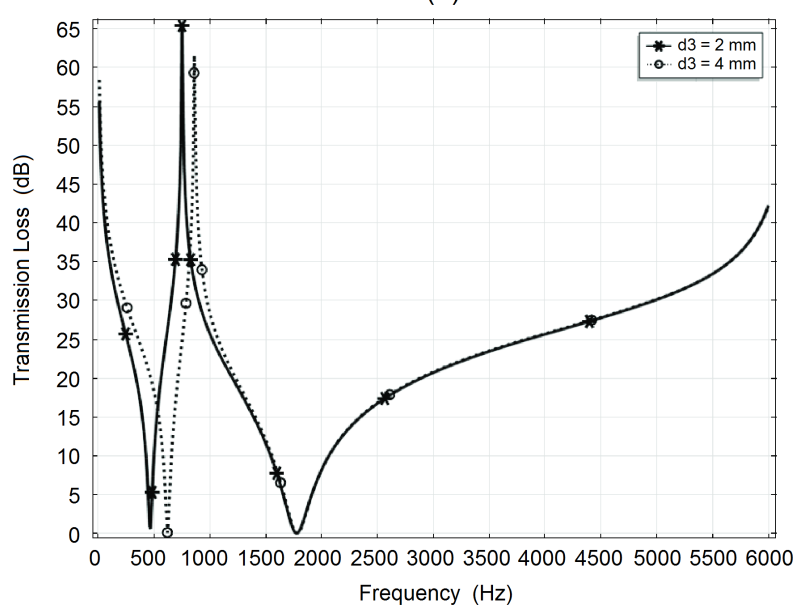

(e)

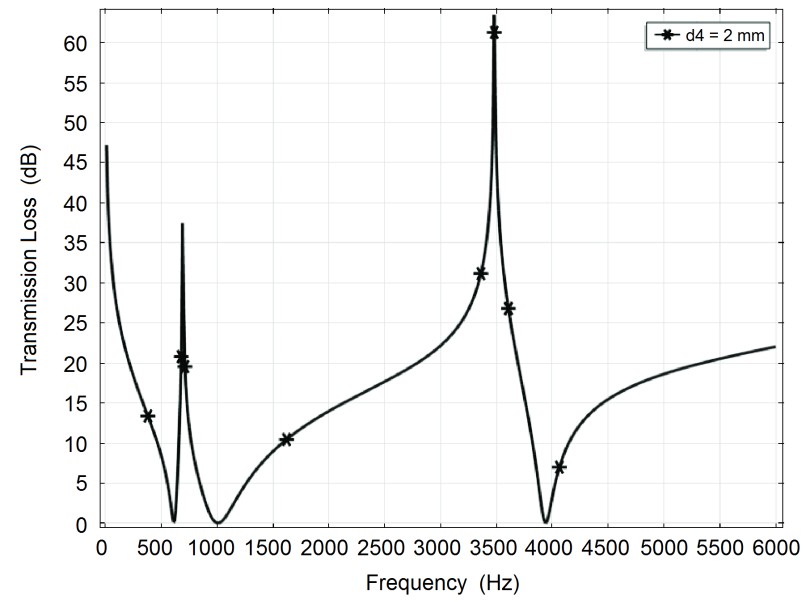

Figure 6. Effect of coaxial ring distribution on transmission loss of membrane-type acoustical metamaterials. 
$0.5 \mathrm{~mm} \times 2 \mathrm{~mm}$, while the two resonance peaks occurred at $480 \mathrm{~Hz}, 5230 \mathrm{~Hz}$ and a single transmission loss peak $89.8 \mathrm{~dB}$ at $3400 \mathrm{~Hz}$ when the cross section of ring is $2 \mathrm{~mm} \times 0.5 \mathrm{~mm}$. Figure 4(d) shows that the transmission loss profile of the triangle cross section ring. The resonance and transmission loss peaks of Fig. 4(d) occur at same frequency with Fig. 4(c) (except the transmission loss peak of Fig. 4(d) higher $9.6 \mathrm{~dB}$ than Fig. 4(c)). This was because the contact stiffness of the triangle and rectangular coaxial ring masses with membrane was close.

\section{THE EFFECT OF THE COAXIAL RING'S MASS ON TRANSMISSION LOSS OF MEMBRANE-TYPE ACOUSTICAL METAMATERIALS}

Through Figs. 4(c) and 4(d), we found that the resonance and transmission loss peaks occurred at same frequency when the coaxial ring's mass, position, and contacting area with the membrane were same. In this section, the transmission loss of membrane-type acoustical metamaterials that have the same contacting area with the membrane will be analyzed, however, it should be noted that the coaxial ring's mass is different. In order to study the influence of coaxial ring's mass on the transmission loss of membrane-type acoustical metamaterials, the transmission loss of membrane-type acoustical metamaterials with different mass coaxial ring were analyzed. We used the example of rectangular cross-section. Figure 5 show that the height of mass block change from $0.2 \mathrm{~mm}$ to $1.0 \mathrm{~mm}$, while the width of mass block is $2 \mathrm{~mm}$ invariable (i.e., the mass of coaxial ring are changed). Through Fig. 5, we found the lowfrequency resonance frequency increased with the decreasing mass, while the high-frequency resonance frequency did not change significantly. Meanwhile, the transmission loss peak frequency moved to a lower frequency with the decreasing mass. When the height of rectangular is $t=1.0 \mathrm{~mm}$, the transmission loss reached a maximum value of $99.7 \mathrm{~dB}$ at $2630 \mathrm{~Hz}$.

\section{THE EFFECT OF COAXIAL RING DISTRIBUTION ON THE TRANSMISSION LOSS OF MEMBRANE-TYPE ACOUSTICAL METAMATERIALS}

In this section, we studied the effect of coaxial ring distribution on transmission loss. We first fixed ring 1 in $r=0 \mathrm{~mm}$ and only let ring 2 change along the radial direction. Figure 6(a) shows that the transmission loss peak frequency of the membrane-type acoustical metamaterials moveed to a higher frequency when the distance of the two rings changed from $d=2 \mathrm{~mm}$ to $d=6 \mathrm{~mm}$. The transmission loss peak magnitude was $117 \mathrm{~dB}$ at $760 \mathrm{~Hz}$ when $d=4 \mathrm{~mm}$. There were two transmission loss peaks at the range from 0 to $6000 \mathrm{~Hz}$ when the distances of the two rings increased to $d=8 \mathrm{~mm}$ or $d=10 \mathrm{~mm}$. Figure 6(a) also shows the transmission loss of the distance for the two rings $d=2,4,10 \mathrm{~mm}$, which exhibited two resonance peaks while there was one resonance peak when $d=6 \mathrm{~mm}$ and three resonance peaks when $d=8 \mathrm{~mm}$ at a range from 0 to $6000 \mathrm{~Hz}$. The transmission loss over a sound level of $20 \mathrm{~dB}$ covered a frequency range of roughly $6000 \mathrm{~Hz}$ when ring was fixed in $d=6 \mathrm{~mm}$. When ring 1 was fixed in $r=2 \mathrm{~mm}$, the distance of two rings change from $2 \mathrm{~mm}$ to $8 \mathrm{~mm}$, the transmission loss profile is shown in Fig. 6(b). We found that the transmission loss over a sound level of $25 \mathrm{~dB}$ was more than a frequency range of $6000 \mathrm{~Hz}$ when the distance of the two rings was $d=6 \mathrm{~mm}$. When ring 1 was fixed $r=4,6,8 \mathrm{~mm}$, the transmission loss profile of ring 2 changed along the radial direction, as shown in Figs. 6(c), 6(d) and 6(e) successively. Figures 6(c) and 6(d) show that the resonance which occurs at the low-frequency and the transmission loss peak frequency when the distance of the two rings increased. Figure 6(e) shows the two rings next to each other at the edge of membrane. The reason that the transmission loss difference caused by coaxial ring distribution may be because that the difference coaxial ring distribution change the stiffness of membrane.

\section{THE ROLES OF MEMBRANE AND RING MASSES ON THE TRANSMISSION LOSS OF MEMBRANE-TYPE ACOUSTICAL METAMATERIALS}

The main roles of membrane and ring masses on the transmission loss of membrane-type acoustical metamaterials are studied in this section. Without the loss of generality, we used the example of rectangular cross section of the ring as $2 \mathrm{~mm} \times$ $0.5 \mathrm{~mm}$ as example. Figure 4(c) shows that there are two resonance peaks at $480 \mathrm{~Hz}, 5230 \mathrm{~Hz}$ and a single transmission loss peak $89.8 \mathrm{~dB}$ at $3400 \mathrm{~Hz}$ when the cross section of the ring was $2 \mathrm{~mm} \times 0.5 \mathrm{~mm}$. The total displacements of the membrane and ring masses at the resonance peaks and transmission loss peak are shown in Fig. 7. Through Fig. 7(a), we found that the membrane and ring masses all left their balance place at the low-frequency resonance frequency $480 \mathrm{~Hz}$. Furthermore, ring 1 and ring 2 vibrated in phase. Figure 7(b) shows that only the center part of the membrane vibrated nearby in a balanced position, the two rings were kept immobile in balanced position at the high-frequency resonance frequency $5230 \mathrm{~Hz}$. At $3400 \mathrm{~Hz}$, the total displacements of membrane and ring masses are shown in Fig. 7(c). Figure 7(d) shows that ring 1 and ring 2 vibrated out of phase at $1200 \mathrm{~Hz}$. This implied that only the membrane played a role at the high frequency, whereas the ring masses affected the low-frequency resonance frequency.

\section{DOUBLE-LEAF MEMBRANE-TYPE ACOUSTICAL METAMATERIALS}

The effects of double-leaf membrane-type acoustical metamaterials are studied in this section. Figure 8 shows the arrangement of membrane-type acoustical metamaterials 1 and 2 , which are parallel to an air-cavity with a depth $D$ between them. When $D=30 \mathrm{~mm}$, the transmission loss of double-leaf membrane-type acoustical metamaterials is shown in Fig. 9. By comparing Fig. 9 and Fig. 4(c), we can find that there are two low-frequency resonance peaks, transmission loss peaks, and high-frequency resonance peaks overlapping each other in the double-leaf membrane-type acoustical metamaterials structure. We can also see that its damping is better than one-leaf membrane-type acoustical metamaterials structure in all frequency region. 


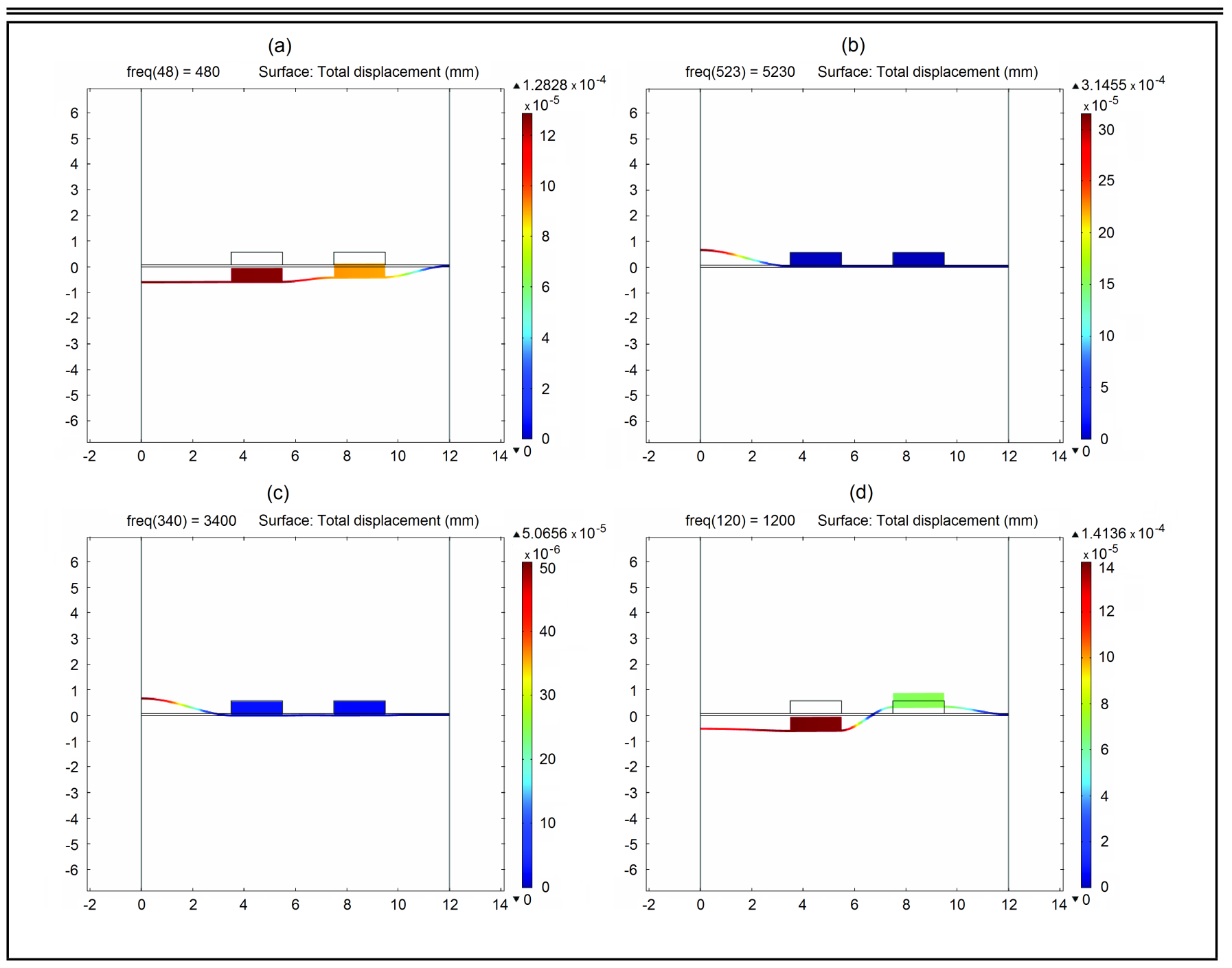

Figure 7. Effects of membrane and ring masses on transmission loss.

To better understand the behavior of the double-leaf structures under excitation, the finite element method was used to predict the resonant mode shapes at discrete frequencies, as seen in Fig. 10. The total displacements of the membrane and ring masses at the resonance peaks and transmission loss peak are shown in Fig. 10. Through Fig. 10(a), we found that the membrane and ring masses of double-leaf structures all left their balance place at the low-frequency resonance frequency $500 \mathrm{~Hz}$. Ring 1 and ring 2 of the double-leaf structures vibrated in phase. Figure 10(a) also shows that the first lay membranetype acoustical metamaterials play a major role at $500 \mathrm{~Hz}$. Figure 10(b) shows that the second lay membrane-type acoustical metamaterials play a major role at $1200 \mathrm{~Hz}$. And ring 1 and ring 2 of the second lay membrane-type acoustical metamaterials vibrated out of phase. Figure 10(c) shows that the center part of the double-leaf membranes vibrated nearby in a balanced position, the four rings masses were kept immobile in a balanced position at $3450 \mathrm{~Hz}$. Figure 10(c) shows that only the center part of the first-leaf membrane vibrated nearby in a balanced position at $5230 \mathrm{~Hz}$.

Furthermore, the effect of the depth $D$ of air-cavity is shown in Fig. 11. According to Fig. 11, the low-frequency resonance frequencies, the transmission loss peaks frequencies, and the high-frequency resonance frequencies varied slightly when the depth $D$ of the air-cavity changed from $20 \mathrm{~mm}$ to $80 \mathrm{~mm}$. But the maximal transmission loss peaks depended on the depth $D$. In addition, there were two resonance peaks between the lowfrequency resonance peaks and the high-frequency resonance peaks when $D=60 \mathrm{~mm}$ and $D=80 \mathrm{~mm}$. There was one resonance peak at a range of $5500 \mathrm{~Hz}$ and $6000 \mathrm{~Hz}$ when $D=$ $20 \mathrm{~mm}$ and $D=80 \mathrm{~mm}$.

\section{CONCLUSIONS}

The present work has been focused on membrane-type acoustical metamaterials of coaxial ring masses. By using the finite element analysis, the transmission loss of membranetype acoustical metamaterials with coaxial ring masses for four kinds of different cross section shape has been studied. The results show that the different contact areas of the coaxial ring with the same mass have influence on the transmission loss peak and the resonance frequencies of membrane-type acoustical metamaterials. Furthermore, the effect of the coaxial ring's mass on transmission loss of membrane-type acoustical metamaterials has been analyzed. We found the low-frequency resonance frequency increase with the mass decreasing while the high-frequency resonance frequency did not change significantly. Meanwhile, the transmission loss peak frequency was moved to a lower frequency with the mass decreasing. In addition, it is also showed that the coaxial ring masses only af- 


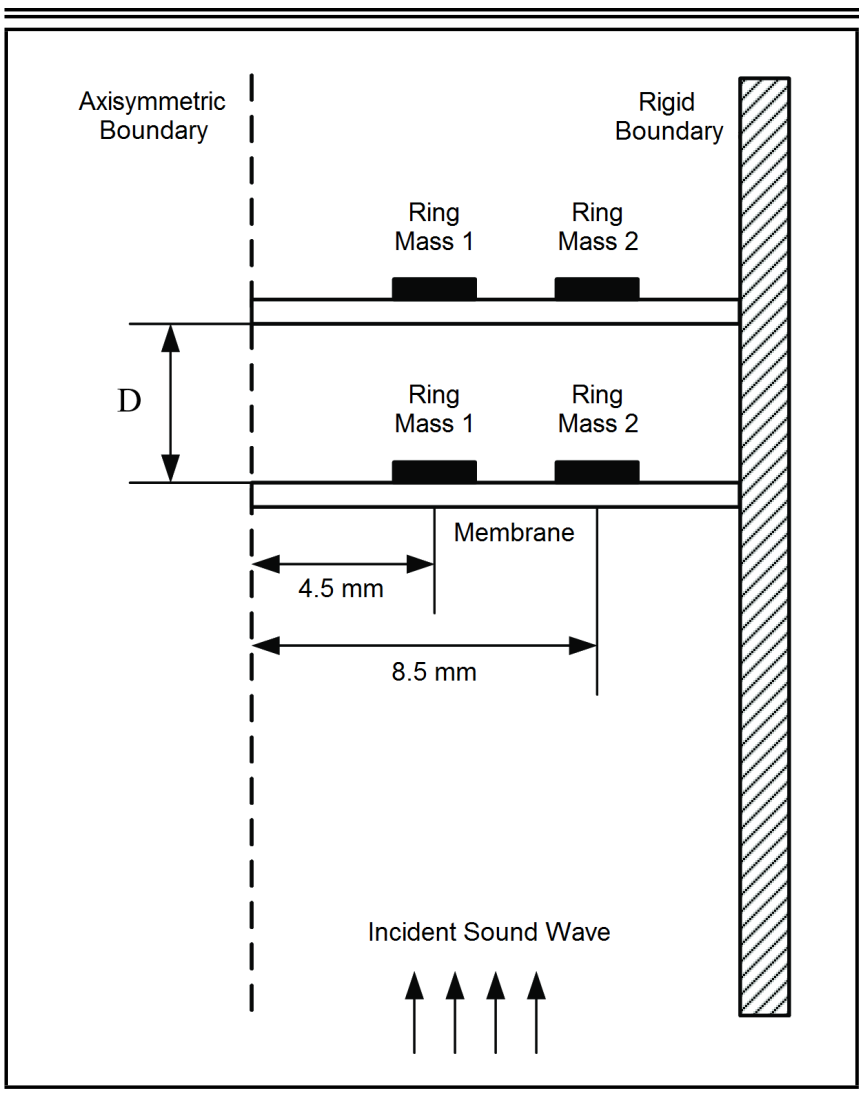

Figure 8. Double-leaf membrane-type acoustical metamaterials finite element analysis models.

fect the TL at the lower frequencies while the membrane is affected at all frequencies. Finally, the effects of the double-leaf membrane-type acoustical metamaterials have been studied. Two low-frequency resonance peaks, transmission loss peaks, and high-frequency resonance peaks that overlap each other have been found in the double-leaf membrane-type acoustical metamaterials structure. And the maximal transmission loss peaks depend on the depth $D$ of air-cavity.

\section{ACKNOWLEDGEMENTS}

This work has been supported by the National Natural Science Foundation of China under Grant No. 11274048, 11604015, and Beijing Natural Science Foundation No.8142016.

\section{REFERENCES}

1 Yang, Z., Dai, H. M., Chan, N. H., Ma, G. C., and Sheng, P. Acoustic metamaterial panels for sound attenuation in the 50-1000 Hz regime, Applied Physics Letters, 96 (4), (2010). http://dx.doi.org/10.1063/1.3299007

2 Bretagne, A., Tourin, A., and Leroy, V. Enhanced and reduced transmission of acoustic waves with bubble meta-screens, Applied Physics Letters, 99, (2011). http://dx.doi.org/10.1063/1.3663623

3 Naify, C. J., Chang, C. M., Knight, G. M., and Nutt, S. Transmission loss and dynamic response of membrane-type locally resonant acoustic metamaterials, Journal of Applied Physics, 108, (2010). http://dx.doi.org/10.1063/1.3514082

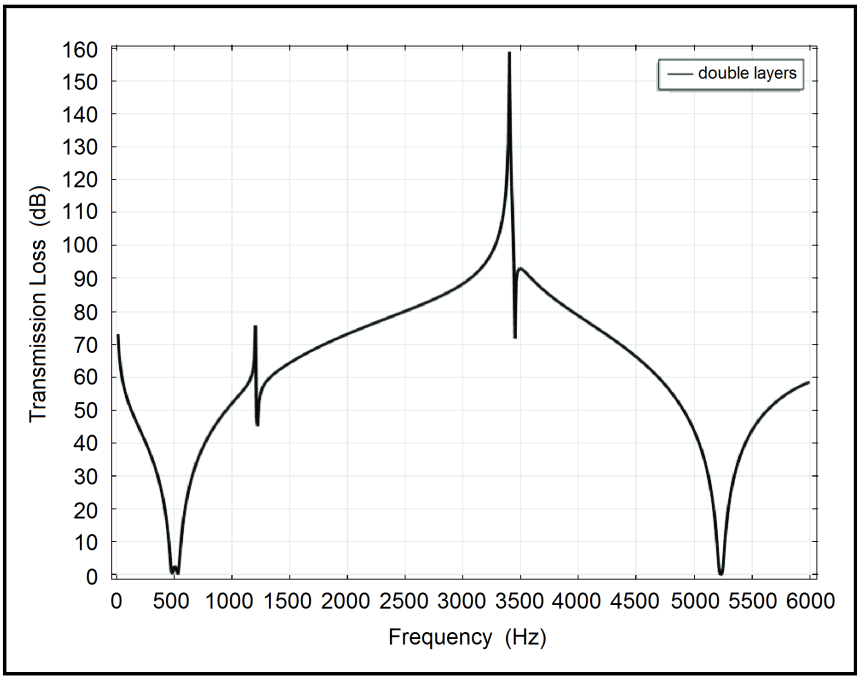

Figure 9. Transmission loss of double-leaf with an air-cavity of depth $D=$ $30 \mathrm{~mm}$.

${ }^{4}$ Fang, N., Lee, H., Sun, C., and Zhang, X. Subdiffraction-limited optical imaging with a silver superlens, Science, 308 (5721), 534-537, (2005). http://dx.doi.org/10.1126/science.1108759

5 Hirsekorn, M. Small-size sonic crystals with strong attenuation bands in the audible frequency range, Applied Physics Letters, 84, (2004). http://dx.doi.org/10.1063/1.1723688

${ }^{6} \mathrm{Li}, \quad$ J. and Chan, C. T. Double-negative acoustic metamaterial, Physical Review E, 70 (5), (2004). http://dx.doi.org/10.1103/PhysRevE.70.055602

7 Lu, M. H., Feng, L., Chen, Y. F. Phononic crystals and acoustic metamaterials, Materials Today, 12 (12), 34-42, (2009). http://dx.doi.org/10.1016/S1369-7021(09)70315-3

8 Ho, K. M., Cheng, C. K., Yang, Z., Zhang, X. X., and Sheng, P. Broadband locally resonant sonic shields, Applied Physics Letters, 83, (2003). http://dx.doi.org/10.1063/1.1637152

9 Liu, Z. Y., Zhang, X. X., Mao, Y. W., Zhu, Y. Y., Yang, Z. Y., Chan, C. T., and Sheng, P. Locally resonant sonic materials, Science, 289 (5485), 1734-1736, (2000). http://dx.doi.org/10.1126/science.289.5485.1734

10 Naify, C. J., Chang, C. M., Knight, G. M., and Nutt, S. Transmission loss of membrane-type acoustic metamaterials with coaxial ring masses, Journal of Applied Physics, 110, (2011). http://dx.doi.org/10.1063/1.3665213

11 Hashimoto, N., Katsura, M., Yasuoka, M., and Fuji, H. Sound insulation of a rectangular thin membrane with additional weights, Applied Acoustics, 33 (1), 21-43, (1991). http://dx.doi.org/10.1016/0003-682X(91)90063-K

12 Hashimoto, N., Katsura, M., and Nishikawa, Y. Experimental study on sound insulation of membranes with small weights for application to membrane structures, Applied Acoustics, 48 (1), 71-84, (1996). http://dx.doi.org/10.1016/0003-682X(95)00062-E 


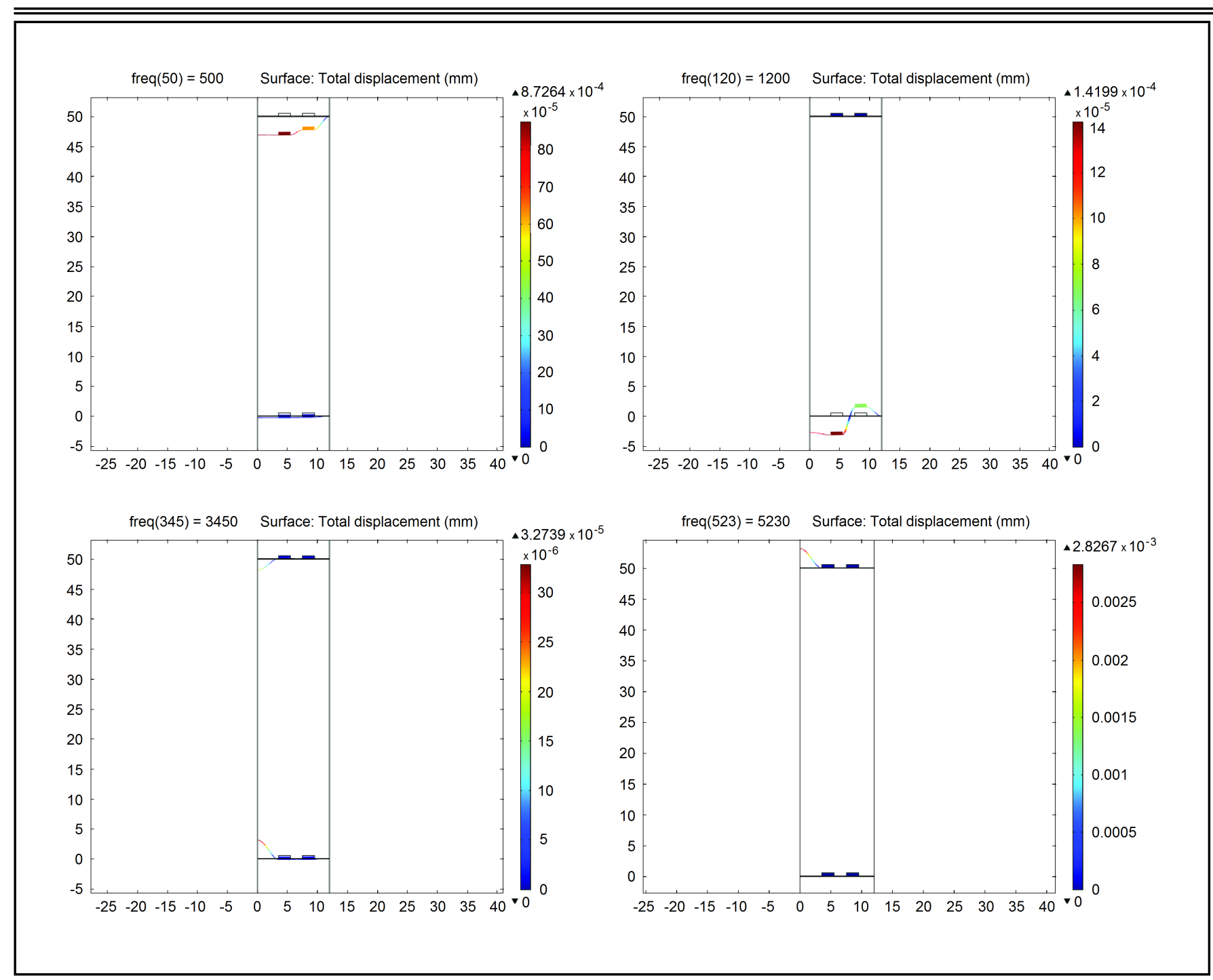

Figure 10. Effects of membrane and ring masses on transmission loss.

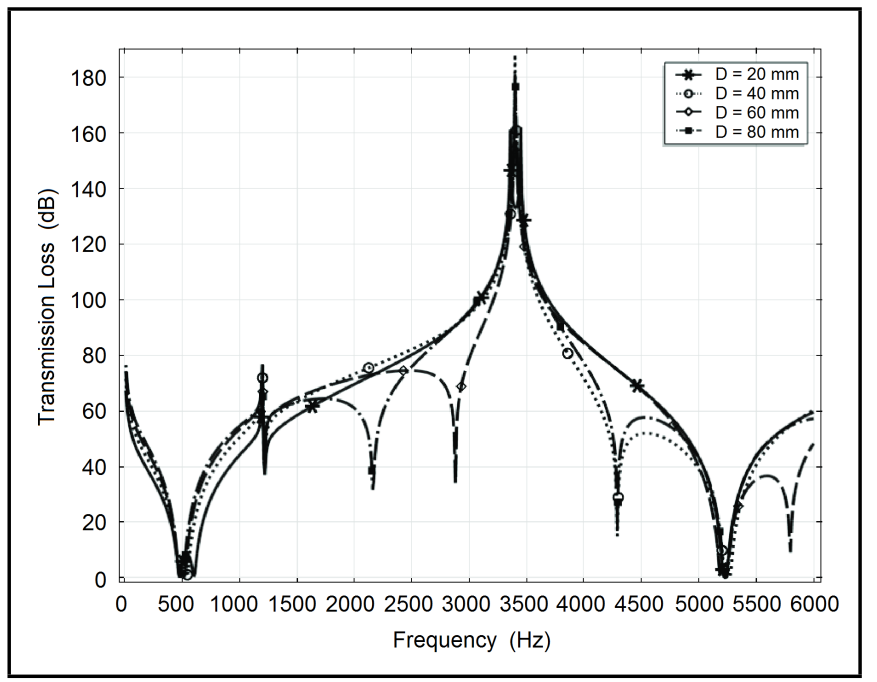

Figure 11. Transmission loss of double-leaf membrane-type acoustical metamaterials with an air-cavity of depth $D=20-80 \mathrm{~mm}$.
13 Yang, Z., Mei, J., Yang, M., Chan, N. H., and Sheng, P. Membrane-type acoustic metamaterial with negative dynamic mass, Physical Review Letters, 101, (2008). http://dx.doi.org/10.1103/PhysRevLett.101.204301

${ }^{14}$ Mei, J., Ma, G., Yang, M., Yang, Z. Y., Wen, W. J., and Sheng, P. Dark acoustic metamaterials as super absorbers for low-frequency sound, Nature Communications, 3, (2012). http://dx.doi.org/10.1038/ ncomms 1758

15 Naify, C. J., Chang, C. M., Knight, G. M., and Scheulen, F. Membrane-type metamaterials: Transmission loss of multicelled arrays, Journal of Applied Physics, 109, (2011). http://dx.doi.org/10.1063/1.3583656

16 Ding, C. L. and Zhao, X. P. Multi-band and broadband acoustics metamaterial with resonant structures, Journal of Physics D : Applied Physics, 44 (21), (2011). http://dx.doi.org/10.1088/0022-3727/44/21/215402 